\title{
Gestational diabetes - modern management and therapeutic approach (Review)
}

\author{
CAMELIA SANDU ${ }^{1}$, CRISTINA BICA $^{1}$, TEODOR SALMEN ${ }^{1}$, ROXANA STOICA ${ }^{2}$, \\ ROXANA BOHILTEA ${ }^{3}$, FLORENTINA GHERGHICEANU ${ }^{4}$, IRINA PACU $^{3}$, SIMONA STEFAN $^{1,2}$, \\ CRISTIAN SERAFINCEANU ${ }^{2,5}$ and ANCA PANTEA STOIAN ${ }^{2}$
}

\author{
${ }^{1}$ National Institute of Diabetes, Nutrition and Metabolic Diseases 'Prof. N.C. Paulescu', \\ 020475 Bucharest; Departments of ${ }^{2}$ Diabetes, Nutrition and Metabolic Diseases, ${ }^{3}$ Obstetrics and Gynecology and \\ ${ }^{4}$ Marketing and Medical Technology, 'Carol Davila' University of Medicine and Pharmacy, 050474 Bucharest; \\ ${ }^{5}$ Department of Hemodialysis, National Institute of Diabetes, Nutrition and Metabolic \\ Diseases 'Prof. N.C. Paulescu', 020475 Bucharest, Romania
}

Received June 17, 2020; Accepted July 17, 2020

DOI: $10.3892 /$ etm.2020.9512

\begin{abstract}
Gestational diabetes mellitus is an important healthcare problem with serious implications both to the mother and to the foetus. The necessity of clear screening criteria for the pregnant woman and also identifying from an early stage the risk groups can be beneficial instruments for better management of gestational diabetes. The present report identify the main screening criteria for patients at risk for gestational diabetes and the therapeutic-nutritional therapy for women that have gestational diabetes. The different diagnostic criteria, as well as the new instruments through which these criteria can be applied, are still heterogeneous, and it is necessary to unify and promote them. The prevalence of gestational diabetes has significantly increased in recent years, and this has led to an increase in the direct and indirect costs of healthcare. Establishing the optimal time and initiating the correct treatment is critical to achieving glycemic control and to minimize the impact on fetal development and perinatal complications.
\end{abstract}

\section{Contents}

1. Introduction

2. Data collection methods

3. Findings of data analysis

Correspondence to: Dr Simona Stefan, National Institute of Diabetes, Nutrition and Metabolic Diseases 'Prof. N.C. Paulescu', 5-7 Ion Movila, 020475 Bucharest, Romania

E-mail: simona_ds2002@yahoo.com

Key words: gestational diabetes mellitus, management of gestational diabetes, screening, risk factors, treatment of gestational diabetes
4. Who should be screened for GDM

5. When to screen

6. Treatment

7. Conclusions

\section{Introduction}

Currently, gestational diabetes mellitus (GDM) is defined as any level of dysglycemia with onset or first acknowledgement during gestation. This current definition remains valid, ignoring the type of management required in order to maintain the glycemic level in the normal range (insulin or national intervention alone). Another important aspect concerning women with GDM is an increased risk for diabetes, especially type 2 diabetes mellitus (T2DM) over the next five or more years following pregnancy (1). The incidence of women with diabetes increased annually by $9 \%$ from 2011 to 2017 , according to a report published in 2020 (2). Temporal pattern of diabetes in pregnant women, which was $6 \%$ greater in women who received antenatal doctor care, $61 \%$ greater in women with hypertension during gestation and $42 \%$ greater in women who had other endocrine diseases (thyroid and pituitary/adrenal diseases) (2). The presence of GDM or other form of diabetes did not affect the risk of obstetrical complications such as postpartum hemorrhage, caesarean section, low neonatal birth weight or special care neonatal intensive care unit admission, after statistical adjustment for demographics and health and care status and behavior (2).

Most obese or overweight women already have an increased risk of developing T2DM; this cluster of patients, especially when becoming pregnant at older ages, face a high risk of developing GDM. There is also an important correlation between the increased prevalence of GDM and the rising prevalence of type 2 diabetes mellitus (T2DM). Contributing further to diabetes in pregnancy is an intra-generational connection between GDM and the risk of newly diagnosed T2DM, which can occur at a fertile age for women. 
It has been well established that indigenous people worldwide experience a heavy burden of the global obesity and T2DM epidemics. They exhibit disparities in diabetes during pregnancy, thus increasing the perinatal morbidity and mortality. The most common intra- and post-pregnancy causes of morbidity-mortality are newborn prematurity, or macrosomia, obstetrical trauma, more frequent indication for caesarean section, congenital anomalies, stillborn or even neonatal death. Women who developed GDM have a high recurrence rate during a future pregnancy, and they remain at high risk of developing T2DM. Concerning the descendants, there is a strong connection between foetal exposure to a hyperglycaemic intra-uterine milieu and their future risk for developing T2DM. Furthermore, the female descendants of GDM mothers have an elevated risk for GDM, in addition to early-onset T2DM. This is a vicious circle that contributes to a female trans-generational diabetes intrapartum risk (3).

\section{Data collection methods}

We searched in the PUBMED the following key words: 'Gestational diabetes', 'gestational diabetes therapy', 'nutrition in gestational diabetes', 'therapeutic management in gestational diabetes', selected several reports and analyzed the main aspects related to the management of gestational diabetes and the medical and dietary therapies.

\section{Findings of data analysis}

Following the search in PUBMED, we selected several reports that were the most relevant for the present investigation: The HAPO study, that compared association of maternal level of glucose and haemoglobin A1C (HbA1C) with adverse outcomes, the HAPO-Follow Up Study that analyzed, 10-14 years later, the children of the mothers that had a 75 g OGT test done during pregnancy and two reports regarding screening and management.

Lowe et al (4) conducted the HAPO Study and enrolled 23,316 participants that performed a $75 \mathrm{~g}$ OGT test at 24-32 weeks of gestation, associated with HbA1C dosing, neonatal anthropometries and cord serum $\mathrm{C}$-peptide value. In previous studies, high concentrations of $\mathrm{HbA} 1 \mathrm{C}$ in pre-existing diabetes has been linked with high risk of development of chronic complications and various adverse events during gestation period such as spontaneous abortion, macrosomia, newborn prematurity or congenital malformations. HbA1C measurements and fructosamine levels are not reliable markers in order to separate women with healthy pregnancy from those with GDM, even though HbA1C levels are lower during pregnancy. The main objective of the HAPO Study was to explain the links between the risk of adverse outcomes associated with maternal dysglycemia less severe than overt diabetes. A $75 \mathrm{~g}$, $2 \mathrm{~h}$ OGT test was performed in a large, international, cohort of women during their third trimester of pregnancy. The primary outcomes were: 'Birth weight $>90$ th percentile, primary caesarean section, clinical neonatal hypoglycemia and level of cord C-peptide $>90$ th percentile'. The secondary outcomes were: 'Preeclampsia, preterm delivery and the sum of skinfolds $>90$ th percentile'. The results gathered from pregnancies in 23,316 women with the levels of glucose values blinded, with birth weight recorded, gestational age determined, and obstetric and neonatal records resulted in 21,064 eligible participants. The relations between $\mathrm{HbA} 1 \mathrm{C}$ and fasting plasma glucose (FPG), 1-h PG, 2-h plasma glucose (PG) and the composite measure were $0.290,0.254,0.227,0.324$ (all $\mathrm{P}<0.001$ ) (4). The maternal HbA1C levels were directly linked with the frequencies of birth weight $>90$ th percentile, primary caesarean section and with the cord serum C-peptide $>90$ th percentile (4). The odds of clinical neonatal hypoglycaemia rose through the first six categories of $\mathrm{HbA} 1 \mathrm{C}$ but were lower in most top groups. The association between HbA1C and the four glucose measurements was significant with all the outcomes. Concerning the birth weight, cord C-peptide, the number of skinfolds and body fat percent $>90$ th percentile, the odds ratios (ORs) for $\mathrm{HbA1C} \%$ were lower than those for PG levels (4). In 2010, The International Association of Diabetes in Pregnancy Study Groups (IADPSG) developed a new diagnostic criteria for GDM. The definition of GDM was based on associations seen between glucose level and birth weight, cord C-peptide level and percent body fat $>90$ th percentile. Macrosomia during pregnancy is an indicator of hyperglycaemia, and it is also linked with foetal hyperinsulinemia and excess adiposity (5).

The HAPO-Follow Up Study included 4160 children aged between 10 and 14 years whose mothers had a $75 \mathrm{~g}$ OGT test at 24-28 weeks of gestation. The study focused on the appearance of impaired glucose tolerance (IGT) and impaired fasting glucose (IFG) in children. Maternal FPG was statistically significantly associated with child FPG, 30-min PG, the sum of the glucose $\mathrm{z}$ scores and $\mathrm{HbA1C}$, but no significance with the 1 and 2-h children PG. On the contrary, maternal 1 and 2-h PG levels during the pregnancy $75 \mathrm{~g}$ OGT test and a maternal sum of 'glucose $\mathrm{z}$ scores' were positively linked with all glucose measures of children and HbA1C (6). The insulin resistance in children, continuous maternal FPG, 1 and 2-h PG and the sum of glucose $\mathrm{z}$ scores were inversely associated with higher insulin resistance. Maternal 1-h PG was reversely associated with the insulinogenic index in children. Maternal FPG and the maternal sum of glucose $\mathrm{z}$ scores during pregnancy were associated with child IFG, while as maternal 1 and 2 h PG and the amount of 'glucose z' scores were associated with IGF. Child adiposity, which is partially dependent on maternal adiposity, can affect insulin sensitivity and glucose metabolism (6).

\section{Who should be screened for GDM}

Previous studies were not definite on whether to perform universal screening or risk-based screening. The American Diabetes Association (ADA) does not recommend testing for GDM low-risk women (7): i) age $<25$ years (7); ii) non-member of an ethnic group (7); iii) body mass index (BMI) $\leq 25 \mathrm{~kg} / \mathrm{m}^{2}$ (7); iv) no history of dysglycemia (7); v) no previous adverse obstetric outcomes (7); vi) without a known family history of diabetes (7).

In 'risk-based' screening, GDM was found in $1.45 \%$ of pregnancies as compared with the universal screening which identified $2.7 \%$ in the same population, demonstrating that 'risk-based' testing missed 50\% of the GDM women (3). According to these results, there is an imperative need for a 
Table I. Primary criteria for the diagnosis of GDM.

\begin{tabular}{|c|c|c|c|c|c|c|c|c|}
\hline Items & Sample & Steps & $\begin{array}{l}\text { OGTT } \\
\text { load }\end{array}$ & $\begin{array}{c}\text { No. } \\
\text { abnormal }\end{array}$ & $\begin{array}{l}\text { Fasting glucose } \\
\mathrm{mg} / \mathrm{dl}(\mathrm{mmol} / \mathrm{l})\end{array}$ & $\begin{array}{l}1 \mathrm{~h} \mathrm{mg} / \mathrm{dl} \\
(\mathrm{mmol} / \mathrm{l})\end{array}$ & $\begin{array}{l}2 \mathrm{~h} \mathrm{mg} / \mathrm{dl} \\
(\mathrm{mmol} / \mathrm{l})\end{array}$ & $\begin{array}{l}3 \mathrm{~h} \mathrm{mg} / \mathrm{dl} \\
(\mathrm{mmol} / \mathrm{l})\end{array}$ \\
\hline O'Sullivan 1964 (11) & B & 2 & $100 \mathrm{~g}$ & $\geq 2$ & $90(5)$ & $165 \quad(9.2)$ & $145(8.1)$ & $125(6.9)$ \\
\hline NDDG 1979 (12) & $\mathrm{P}$ & 2 & $100 \mathrm{~g}$ & $\geq 2$ & $105(5.8)$ & $190(10.6)$ & $165(9.2)$ & $145(8.0)$ \\
\hline C\&C 1982 (13) & $\mathrm{P}$ & 2 & $100 \mathrm{~g}$ & $\geq 2$ & $95(5.3)$ & $180(10)$ & $155(8.6)$ & $140(7.8)$ \\
\hline EASD 1996 (14) & $\mathrm{P}$ & 1 & $75 \mathrm{~g}$ & $\geq 1$ & $108(6)$ & $\mathrm{X}$ & $162(9)$ & $\mathrm{X}$ \\
\hline ADIPS 1998 (15) & $\mathrm{P}$ & 1 & $75 \mathrm{~g}$ & $\geq 1$ & $100(5.5)$ & $\mathrm{X}$ & $144(8)$ & $\mathrm{X}$ \\
\hline WHO 1999 (16) & $\mathrm{P}$ & 1 & $75 \mathrm{~g}$ & $\geq 1$ & $126(7)$ & $X$ & $140(7.8)$ & $\mathrm{X}$ \\
\hline IADPSG 2010 (5) & $\mathrm{P}$ & 1 & $75 \mathrm{~g}$ & $\geq 1$ & $92(5.1)$ & $180(10)$ & $153(8.5)$ & $\mathrm{X}$ \\
\hline
\end{tabular}

X, not applicable; B, whole blood; P, plasma; NDDG, National Diabetes Data Group; C\&C, Carpenter and Coustan; EASD, European Association for Study Diabetes; ADIPS, Australian Diabetes in Pregnancy Society; WHO, World Health Organization; IADPSG, International Association of Diabetes and Pregnancy Study Groups.

Table II. One step and Two-step methods for diagnoses GDM.

\begin{tabular}{lcc}
\hline Items & One step procedure $(75 \mathrm{~g} \mathrm{OGTT})(16,20)$ & Two-step procedure $(50 \mathrm{~g}$ OGTT) $(17,20)$ \\
\hline Fasting serum glucose & $92 \mathrm{mg} / \mathrm{dl}(5.1 \mathrm{mmol} / \mathrm{l})$ & $95 \mathrm{mg} / \mathrm{dl} \mathrm{or} 105 \mathrm{mg} / \mathrm{dl}(5.5 / 5.8 \mathrm{mmol} / \mathrm{l})$ \\
1-h serum glucose & $180 \mathrm{mg} / \mathrm{dl}(10.0 \mathrm{mmol} / \mathrm{l})$ & $180 \mathrm{mg} / \mathrm{dl} \mathrm{or} 190 \mathrm{mg} / \mathrm{dl}(10.0 / 10.6 \mathrm{mmol} / \mathrm{l})$ \\
2-h serum glucose & $153 \mathrm{mg} / \mathrm{dl}(8.5 \mathrm{mmol} / \mathrm{l})$ & $155 \mathrm{mg} / \mathrm{dl} \mathrm{or} 165 \mathrm{mg} / \mathrm{dl}(8.6 / 9.2 \mathrm{mmol} / \mathrm{l})$ \\
3-h serum glucose & & $145 \mathrm{mg} / \mathrm{dl}(7.8 / 8.0 \mathrm{mmol} / \mathrm{l})$ \\
\hline
\end{tabular}

OGTT, oral glucose tolerance test.

universal screening tool, especially in the Southeast Asian population such as in Indian women who have an elevated prevalence of T2DM and a genetic susceptibility for GDM (8-10).

\section{When to screen}

Generally, the current screening for GDM is recommended between 24-28 weeks of pregnancy. This period was determined because the level of the insulin resistance is increased in the second trimester, and glucose levels are rising because the insulin secretion is inadequate to compensate for this resistance $(8,9)$.

On the contrary, 24-28-week window was chosen because the level of placental hormones is higher, causing secondary insulin resistance, which increases the risk of GDM as the pregnancy advances. Testing for GDM before the second trimester may not identify all GDM patients. However, performing tests too late, in the third trimester, does not allow enough time for the metabolic interventions. Therefore, the general recommendation is to perform the test in the usual window of 24-28 weeks of pregnancy. The International Association of Diabetes and Pregnancy Study Group (IADPSG), also approved by ADA based on HAPO study, the indication is to perform on the first prenatal visit the following blood tests: FPG, HbA1C or even a random plasma glucose in all pregnant women. If the results are not a positive for an overt DM and FPG is higher than $92 \mathrm{mg} / \mathrm{dl}$, diagnosis of GDM is definite. If fasting glucose is 'lower than $92 \mathrm{mg} / \mathrm{dl}$ at the first antenatal visit, it is recommended to perform a 2-h $75 \mathrm{~g}$ OGT test at 24-28 weeks' $(8,9)$. There is no consensus so far in the diagnosis of gestational diabetes, but the guidelines are listed in Table I (5,11-16).

There are two different methods proposed by ADA in 2015 for the diagnosis of GDM in women without diagnosed diabetes before pregnancy, both techniques are shown in Table II, where a positive diagnosis is defined if PG values equal or exceed the limit (17).

First is the 'One Step Procedure': The 75 g OGT test is performed in the morning, following fasting $>8 \mathrm{~h}$ with $\mathrm{PG}$ measurement fasting, 1 and 2-h at 24-28 weeks of gestation in women not having pre-existent diabetes $(7,17)$.

The 'Two-Step Procedure': Step one consists in performing the 50-g glucose challenge test, irrespective of the last meal, at 24-28 weeks in women not having pre-existing diabetes. If PG at 1-h after the load is $>140 \mathrm{mg} / \mathrm{dl}$ ( $7.8 \mathrm{mmol} / \mathrm{l})$, step two will follow. A 100-g glucose test should be performed $(7,17,18)$. Perinatal outcomes in the HAPO study when IADPSG criteria for GDM are applied as shown in Table III.

Of great interest is the development of easy to apply, efficient and inexpensive screening tools. Another method of screening taken into account is visceral adipose tissue depth in early pregnancy. Some studies have suggested that it is possible to predict GDM early in the pregnancy by monitoring the visceral adipose tissue depth (VAD). VAD is described as a secure and easy method measured by ultrasound in early pregnancy, by Alves et al (19). A prospective study was made, in which VAD was estimated at $\sim 14$ weeks of pregnancy, while an OGTT was performed at 24-28 weeks, according to the IADPSG criteria. 
Table III. The perinatal outcomes from the HAPO study (4).

\begin{tabular}{|c|c|c|c|}
\hline Outcome & $\begin{array}{c}\text { Frequency in } \\
\text { GDM }(\%)\end{array}$ & $\begin{array}{c}\text { Frequency in } \\
\text { non-GDM }(\%)\end{array}$ & $\begin{array}{c}\text { Frequency } \\
\text { difference }(\%)\end{array}$ \\
\hline Pre-eclampsia & 9.1 & 4.5 & 4.6 \\
\hline Delivery at $<37$ weeks & 9.4 & 6.4 & 3.0 \\
\hline Primary caesarean delivery & 24.4 & 16.8 & 7.6 \\
\hline Shoulder dystocia or birth injury & 1.8 & 1.3 & 0.5 \\
\hline Intensive neonatal care & 9.1 & 7.8 & 1.3 \\
\hline Clinical neonatal hypoglycaemia & 2.7 & 1.9 & 0.8 \\
\hline Neonatal hyperbilirubinemia & 10.0 & 8.0 & 2.0 \\
\hline Birthweight $>90$ th percentile & 16.2 & 8.3 & 7.9 \\
\hline Cord C-peptide $>90$ th percentile & 17.5 & 6.7 & 10.8 \\
\hline Percent body adipose tissue content $>90$ th percentile & 16.6 & 8.5 & 8.1 \\
\hline
\end{tabular}

GDM, gestational diabetes mellitus.

Logistic regression analysis was used to estimate the predictive value of VAD, above and beyond pre-pregnancy BMI. Of the total 627 pregnant women enrolled, 518 women completed the study. VAD was assessed at a mean of 14.4 weeks of pregnancy. In total 87 women (16.8\%) developed GDM. The odds ratio (OR) for developing GDM was 1.99 (95\% CI, 1.59-2.46) per $1-\mathrm{cm}$ increase in VAD. The ROC under the curve for developing GDM was higher for VAD (0.70) than for pre-pregnancy BMI (0.57). They concluded that a higher VAD level may better predict GDM than pre-pregnancy BMI, and measuring VAD could become a useful screening tool for GDM (19).

Another case-control study conducted by Lovati et al (20) evaluated the level of pregnancy-associated plasma protein-A (PAPP-A) and its correlation with GDM for predicting GDM in primiparous women. The authors used data from 307 pregnant women with GDM and 366 control healthy pregnant women. A statistic model was used to analyze the association between first trimester data and subsequent clinical outcomes. The two groups (gestational diabetic and control group) were significantly statistically different regarding the age $(\mathrm{P}<0.001)$, BMI $(\mathrm{P}<0.001)$, weight $(\mathrm{P}<0.001)$, family history of diabetes $(\mathrm{P}<0.001)$, PAPP-A levels and PAPP-A corrected multiple of the median $(\mathrm{MoM})(\mathrm{P}<0.001)(20)$. The conclusions were that low PAPP-A is incorrectly linked with GDM, and when insulin therapy was used the PAPP-A values were lower. The authors concluded that dosing the PAPP-A to first-trimester screening could provide a more accurate prediction of women at high risk of developing GDM (20).

The study conducted by D'Ambrosi et al (21) compared maternal subcutaneous tissue and the visceral adipose thickness between women with GDM and nondiabetic pregnancies. Adipose thickness was evaluated performing a transabdominal ultrasound between 24-28 weeks of gestation. The women underwent to $75 \mathrm{~g}$ OGT test as a diagnostic test for GDM. In total, 56 women with a positive glucose alteration test and 112 nondiabetic pregnancies were included in the survey. Ultrasound evaluations of subcutaneous and visceral adipose tissues were developed into multiples of the median (MoM), adjusted for gestational age. The mean of MoM in GDM women was significant compared with healthy pregnancies (1.31 vs. $1.07 ; \mathrm{P}=0.011)(21)$.

Likewise, the mean visceral thickness (MoM) was more significant in women diagnosed with GDM compared with controls (1.61 vs. 1.06; $\mathrm{P}<0.001)(21)$. The study demonstrated that visceral adipose thickness is significantly and independently associated with GDM. Also, the sonographic thickness of maternal visceral adipose tissue was higher in women with GDM compared with control pregnancies, of all other known risk factors associated with GDM (21).

Another enlightening study by Cisneiros et al (22) showed that for each cm increase in VAT depth, there is a $206 \mathrm{~g}$ (95\% CI, 101-311) increase in mean birth weight. VAT depth and the other model covariates together explained more of the variance in birth weight $(\mathrm{P}<0.001)$ than pre-pregnancy BMI with the other covariates in the same model $(\mathrm{P}=0.076)$. They performed three glucose tolerance test measures, between 36-39 weeks of gestation. The VAT level in the first half of pregnancy can predict newborn weight better than BMI value, but it is not associated with glucose tolerance in late pregnancy (22).

\section{Treatment}

The majority of women achieve glycemic targets with nutritional therapy alone, and $\sim 30 \%$ will require supplementary treatment. The appropriate management of gestational diabetes is self-blood glucose monitoring, medical nutritional therapy and insulin therapy when target blood glucose levels are not met with medical nutritional intervention alone. Pre-pregnancy weight loss and normalization of body mass index can improve future pregnancy outcome. As a practical matter, once pregnancy occurs, pharmacologic and surgical interventions are not used for treating obesity and efforts to limit gestational weight gain have met with limited success; however, gestational diabetes can be identified, diagnosed and well treated. A proper diabetes management can prevent adverse outcomes as well as decrease gestational weight gain (23). 
The ADA states that nutrition therapy for gestational diabetes mellitus provides adequate nutrition to promote foetal and maternal well-being while achieving normoglycemia with an absence of ketosis and provides adequate energy levels for appropriate weight gain in pregnancy. The nutritional therapy begins with a nutrition assessment exam accordingly with the Dietary Reference Intakes (DRI) for all pregnant women (minimum of $175 \mathrm{~g}$ of carbohydrate, $71 \mathrm{~g}$ of protein, and $28 \mathrm{~g}$ of fiber) (18). Usually, women require between 1,800-2,500 kcal/day. If the women have normal BMI (BMI, 18.5-24.9 kg/m²) during pregnancy, reasonable caloric intake is $30 \mathrm{kcal} / \mathrm{kg} / \mathrm{day}$; when they are overweight (BMI, 25.0-29.9 kg/m²) or obese (BMI, 30.0-39.9 kg/m²), reasonable caloric intake is $22-25 \mathrm{kcal} / \mathrm{kg} /$ day $(7,18)$.

For morbidly obese women with a BMI $>40 \mathrm{~kg} / \mathrm{m}^{2}$, the reasonable caloric intake is $12-14 \mathrm{kcal} / \mathrm{kg} / \mathrm{day}$ (present pregnant weight), but it is prudent for obese women to consume a minimum of $1,800 \mathrm{kcal} /$ day to prevent ketosis (7). For underweight women $\left(\mathrm{BMI}<18.5 \mathrm{~kg} / \mathrm{m}^{2}\right)$, reasonable caloric intake is $35-40 \mathrm{kcal} / \mathrm{kg} /$ day to achieve recommended weight gain, blood glucose goals, and nutrient intake. It is recommended to achieve the total number of calories from carbohydrates ( $\sim 40 \%$ of total calories intake), proteins ( $20 \%$ of total calories intake) and fats (40\% of total calories; saturated fat intake should be $<7 \%$ of total calories intake) (7). Protein intake should be included in all meals and snacks, because they promote satiety, slow the absorption of carbohydrates into the bloodstream, and provide adequate calories. A bedtime high-protein snack is sometimes needed in order to prevent accelerated overnight ketosis process (7).

The optimal approach to glucose monitoring has not been determined. When initially diagnosed with gestational diabetes mellitus, we ask patients to keep a journal of their blood glucose levels (fasting and one or two hours after the first bite of each meal). Multiple daily measurements are the key to recognizing women who should begin treatment with an antihyperglycemic agent. The findings should be recorded in a glucose log register, along with dietary information. This facilitates the identification of glycaemic patterns and helps immeasurably in interpreting results stored in the memory of modern records.

The ADA and American College of Obstetricians and Gynecologists (ACOG) recommended glucose targets are: i) FG level $<95 \mathrm{mg} / \mathrm{dl}(5.3 \mathrm{mmol} / \mathrm{l})(7,17,18)$. ii) One-hour PG $<140 \mathrm{mg} / \mathrm{dl}$ (7.8 mmol/l) $(7,17,18)$. iii) Two-hour postprandial glucose level $<120 \mathrm{mg} / \mathrm{dl}(6.7 \mathrm{mmol} / \mathrm{l})(7,17,18)$. We initiate therapy when $>30 \%$ of the blood glucose values are above the previously mentioned thresholds $(7,12,17,18,24)$.

Two pharmacologic options are available in pregnant women who need to better control their blood glucose: Different insulin regimens (several insulin analogues) and several selected antihyperglycemic oral agents (such as metformin or glyburide), along with medical and nutritional intervention. We consider insulin the first-line of medical treatment and believe that oral antihyperglycemic agents represent an alternative when nutritional therapy fails, and women decline or are unable to safely manage insulin therapy. Both ACOG (18) and ADA $(7,17)$ prefer the use of different insulin regimens for the therapeutical management of diabetes during pregnancy, but have endorsed the use of oral antihyperglycemic agents (metformin/glyburide) in certain circumstances. In the United States, oral antihyperglycemic agents have not been recommended or approved for the treatment of gestational diabetes mellitus by the US Food and Drug Administration (FDA). ACOG recommends an oral agent for women who decline insulin therapy or when the patient will be unable to afford or safely administer insulin and recommends metformin over glyburide as the preferred oral antihyperglycemic agent (1). They emphasize that patients should understand the limitations of available safety data and the possibility that insulin supplementation may be necessary to achieve euglycemia. The ADA also states that metformin should be avoided in case of hypertension, preeclampsia, or in case of risk for lower intrauterine growth (16-18).

\section{Conclusions}

The most important step to proper management of patients with gestational diabetes is to unify all the screening methods because each one has an essential contribution to a thorough evaluation (25). Another important subject is having a multidisciplinary team composed of physicians specialized in diabetes that work together with gynecologists for intervening efficiently.

The above studies pointed out the necessity of universal screening because only half of the GDM is diagnosed through risk screening. The adverse events on the foetus were positively correlated with the A1c level of the mother, demonstrating that Alc testing has some advantages, associating it with the OGTT testing at 24-28 weeks may be a useful tool for correctly diagnosing GDM.

The first step in treating GDM is diet and in many cases the only one necessary, but if $30 \%$ of the targeted plasma glucose levels are exceeded (FPG>95 mg/dl, 1-h PG over $140 \mathrm{mg} / \mathrm{dl}$, 2-h PG over $120 \mathrm{mg} / \mathrm{dl}$ ) it is a first criteria for introducing pharmacotherapy, insulin being the first choice and the only one approved by the FDA. The last vital step that must not be missed is screening the mother at 6-8 weeks postpartum for type 2 diabetes.

\section{Acknowledgements}

Not applicable.

\section{Funding}

No funding was received.

\section{Availability of data and materials}

Data sharing is not applicable to this article, as no datasets were generated or analyzed during the current study.

\section{Authors' contributions}

APS and CSa conceived the study. CB, TS, FG, IP and RS acquired the data and performed the literature research. RB, SS and CSe revised the study for important intellectual content. All authors were involved in the writing of the manuscript. All authors read and approved the final version of the manuscript. 


\section{Ethics approval and consent to participate}

Not applicable.

\section{Patient consent for publication}

Not applicable.

\section{Competing interests}

APS is currently Vice President of the Romanian National Diabetes Committee and has given talks, attended conferences and participated in advisory boards sponsored by various pharmaceutical companies. The other authors declare that they have no competing interests.

\section{References}

1. Mpondo BC, Ernest A and Dee HE: Gestational diabetes mellitus: Challenges in diagnosis and management. J Diabetes Metab Disord 14: 42, 2015.

2. Khajehei $\mathrm{M}$ and Assareh $\mathrm{H}$ : Temporal trend of diabetes in pregnant women and its association with birth outcomes, 2011 to 2017. J Diabetes Complications 34: 107550, 2020.

3. Dyck RF, Karunanayake C, Pahwa P, Stang M and Osgood ND: Epidemiology of diabetes in pregnancy among first nations and non-first nations women in Saskatchewan, 1980-2013. Part 2 : Predictors and early complications; Results from the diabetes in pregnancy: Outcomes and ramification from a retrospective longitudinal intra-intergenerational study in Saskatchewan Project. Can J Diabetes: Nov 20, 2019 (Epub ahead of print). doi: https://doi.org/10.1016/j.jcjd.2019.11.001

4. Lowe LP, Metzger BE, Dyer AR, Lowe J, McCance DR, Lappin TR, Trimble ER, Coustan DR, Hadden DR, Hod M, et al: HAPO Study cooperative research group: Hyperglycemia and Adverse Pregnancy Outcome (HAPO) study: Associations of maternal A1C and glucose with pregnancy outcomes. Diabetes Care 35: 574-580, 2012.

5. Metzger BE, Gabbe SG, Persson B, Buchanan TA, Catalano PA, Damm P, Dyer AR, Leiva A, Hod M, Kitzmiller JL, et al: International association of diabetes and pregnancy study groups consensus panel: International association of diabetes and pregnancy study groups recommendations on the diagnosis and classification of hyperglycemia in pregnancy. Diabetes Care 33 676-682, 2010.

6. Scholtens DM, Kuang A, Lowe LP, Hamilton J, Lawrence JM, Lebenthal Y, Brickman WJ, Clayton P, Ma RC, McCance D, et al: HAPO Follow-up study cooperative research group: Hyperglycemia and adverse pregnancy outcome follow-up study (HAPO FUS): Maternal glycemia and childhood glucose metabolism. Diabetes Care 42: 381-392, 2019.

7. American Diabetes Association: 14. Management of diabetes in pregnancy: Standards of medical care in diabetes-2020. Diabetes Care 43 (Suppl 1): S183-S192, 2020.

8. Brown FM and Wyckoff J: Application of one-step IADPSG versus two-step diagnostic criteria for gestational diabetes in the real world: Impact on health services, clinical care, and outcomes. Curr Diab Rep 17: 85, 2017.
9. Rani PR and Begum J: Screening and diagnosis of gestational diabetes mellitus, where do we stand. J Clin Diagn Res 10: QE01-QE04, 2016.

10. Buchanan TA, Xiang AH and Page KA: Gestational diabetes mellitus: Risks and management during and after pregnancy. Nat Rev Endocrinol 8: 639-649, 2012.

11. O'Sullivan JB and Mahan CM: Criteria for the oral glucose tolerance test in pregnancy. Diabetes 13: 278-285, 1964.

12. Classification and diagnosis of diabetes mellitus and other categories of glucose intolerance. National Diabetes Data Group. Diabetes 28: 1039-1057, 1979.

13. Carpenter MW and Coustan DR: Criteria for screening tests for gestational diabetes. Am J Obstet Gynecol 144: 768-773, 1982.

14. Brown CJ, Dawson A, Dodds R, Gamsu H, Gillmer M, Hall M, Hounsome B, Knopfler A, Ostler J, Peacock I, et al: Report of the pregnancy and neonatal care group. Diabet Med 13 (Suppl 4): S43-S53, 1996.

15. Diagnosis of Gestational Diabetes Mellitus (GDM) in Australia: College Communique, [14/10/2014]. https://www.ranzcog. edu. au/womens-health/collegecommuniques/1392-diagnosis-of-gestational-diabetes-mellitusgdm-in-australia.html. Last updated on November 2014. Accessed, January, 2020.

16. Definition, Diagnosis and Classification of Diabetes Mellitus and its Complications. World Health Organization, Geneva, pp19-20, 1999.

17. American Diabetes Association: Management of diabetes in pregnancy: Standards of medical care in diabetes-2015. Diabetes Care 38 (Suppl 1): S77-S80, 2015.

18. Committee on practice bulletins-obstetrics: ACOG Practice Bulletin No. 190: Gestational diabetes mellitus. Obstet Gynecol 131: e49-e64, 2018.

19. Alves JG, Souza ASR, Figueiroa JN, de Araújo CAL, Guimarães A and Ray JG: Visceral adipose tissue depth in early pregnancy and gestational diabetes mellitus - a cohort study. Sci Rep 10: 2032, 2020.

20. Lovati E, Beneventi F, Simonetta M, Laneri M, Quarleri L, Scudeller L, Albonico G, Locatelli E, Cavagnoli C, Tinelli C, et al: Gestational diabetes mellitus: Including serum pregnancy-associated plasma protein-A testing in the clinical management of primiparous women? A case-control study. Diabetes Res Clin Pract 100: 340-347, 2013.

21. D'Ambrosi F, Crovetto F, Colosi E, Fabietti I, Carbone F, Tassis B, Motta S, Bulfoni A, Fedele L, Rossi G and Persico N: Maternal subcutaneous and visceral adipose ultrasound thickness in women with gestational diabetes mellitus at 24-28 weeks' gestation. Fetal Diagn Ther 43: 143-147, 2018.

22. Cisneiros RM, Dutra LP, Silveira FJ, Souza AR, Marques M, Amorim MM, Urquia ML, Ray JG and Alves JG: Visceral adiposity in the first half of pregnancy predicts newborn weight among adolescent mothers. J Obstet Gynaecol Can 35: 704-709, 2013.

23. Ionescu CA, Dimitriu M, Poenaru E, Bănacu M, Furău GO, Navolan D and Ples L: Defensive caesarean section: A reality and a recommended health care improvement for Romanian obstetrics. J Eval Clin Pract 25: 111-116, 2019.

24. Durnwald C, Nathan DM, Werner EF and Barss VA: Gestational diabetes mellitus: Glycemic control and maternal prognosis. https://www.uptodate.com/contents/gestational-diabetes-mellitusglycemic-control-and-maternal-prognosis, 2020.

25. Agarwal MM: Consensus in gestational diabetes mellitus: Looking for the Holy Grail. J Clin Med 7: 123, 2018. 